\title{
LIDERAZGO PEDAGÓGICO DEL DIRECTOR Y EVALUACIÓN DEL DESEMPEÑO DOCENTE EN LAS INSTITUCIONES EDUCATIVAS DEL NIVEL SECUNDARIO DE LA RED N 09 DEL DISTRITO DE VILLA MARÍA DEL TRIUNFO, LIMA 2014
}

\begin{abstract}
EDUCATIONAL LEADERSHIP OF DIRECTOR AND TEACHER PERFORMANCE EVALUATION OF EDUCATIONAL INSTITUTIONS OF SECONDARY NETWORK No.
\end{abstract} 09 OF Villa María del Triunfo, LIMA 2014.

Zoila Ayvar Bazán 1

\section{RESUMEN}

La presente investigación es de tipo básica, y las variables de estudio son, el liderazgo pedagógico del director y la evaluación del desempeño docente. El estudio se llevó a cabo en las instituciones educativas del nivel secundario de la RED $N^{\circ} 09$, del distrito de Villa María del Triunfo, Lima, Perú. La unidad de estudio está conformada por 4 directivos y 200 docentes y la muestra está constituida por 4 directivos y 132 docentes.

El diseño de investigación es transaccional, correlacional causal. Para la recolección de datos se elaboró dos cuestionarios: para el liderazgo pedagógico del director y para el desempeño docente, que se aplicó a los 4 directivos y 132 docentes de la muestra.

Los instrumentos aplicados fueron sometidos a juicio de expertos y cumplen con las cualidades de validez y confiabilidad. El estudio plantea la siguiente hipótesis general, el liderazgo pedagógico del director influye significativamente en la evaluación del desempeño docente en las instituciones educativas del nivel secundario de la RED $\mathrm{N}^{\circ} 09$, del distrito de Villa María del Triunfo, Lima 2014.

Los resultados obtenidos nos permiten rechazar la hipótesis nula y aceptar la hipótesis alterna, pues se ha observado a través de la prueba de Kruskal-Wallis la influencia significativa entre el liderazgo pedagógico y el desempeño docente con un nivel de significancia del 95\% obteniendo un p_valor de 0.00; además, se aplicó la prueba de regresión lineal que permite observar la influencia del liderazgo pedagógico en un 42,4\% en el desempeño docente.

\footnotetext{
1 Magíster (c) en Educación con mención en Evaluación y Acreditación de la Calidad Educativa en la Universidad Nacional Mayor de San Marcos.
} 
Palabras clave: Liderazgo pedagógico del director y evaluación del desempeño docente.

\section{ABSTRACT}

This research is basic type, the variables of study, educational leadership of the director and the evaluation of the teaching performance. The study was carried out in the educational institutions of the secondary level of the NETWORK $\mathrm{N}^{\circ} 09$, district of Villa Maria del Triunfo, Lima, Peru. The unit of study is formed by 4 managers and 200 teachers and it shows is constituted by 4 managers and 132 teachers.

Research design is transactional, correlation causal. For the collection of data was prepared two questionnaires: for pedagogical leadership from the director and teacher performance, which was applied to 4 managers and 132 teachers in the sample.

Applied instruments were subjected to expert opinion and comply with the qualities of validity and reliability. The study proposes the following general hypothesis, the pedagogical leadership from the director significantly influence the evaluation of the teaching performance in the educational institutions of the secondary level of the NETWORK $\mathrm{N}^{\circ} 09$, district of Villa Maria del Triunfo, Lima 2014.

The results obtained allow us to reject the null hypothesis and accept the alternative hypothesis, as he has been observed through the Kruskal-Wallis test significant influence between the pedagogical leadership and teaching performance with a significance level of $95 \%$ with a $\mathrm{p}$ value of 0.00 ; in addition, applied the test of linear regression that allows to observe the influence of the educational leadership by $42.4 \%$ in the teaching performance

KEY WORDS: leadership teaching of the director and evaluation of the performance teaching.

\section{INTRODUCCIÓN}

La Educación Básica Regular EBR, de gestión pública, nivel secundario, en el Perú se encuentra en una situación crítica. Los estándares de medición y evaluación de la calidad educativa demuestran que los niños y los adolescentes no logran las competencias básicas en las áreas de comunicación integral y el desarrollo del pensamiento lógico.

Esta situación crítica de la calidad de educación que brinda nuestro sistema 
educativo, se ha evidenciado en los resultados obtenidos en las evaluaciones censales y muéstrales, llevados a cabo por organismos nacionales e internacionales, como la Evaluación Nacional de Rendimiento Estudiantil y la Evaluación Censal de Estudiantes (ECE) del Ministerio de Educación; el Primer Estudio Internacional Comparativo (PEIC), y el Segundo Estudio Regional Comparativo y Explicativo (SERCE) del Laboratorio Latinoamericano de Evaluación de la Calidad de la Educación (LLECE), auspiciado por la UNESCO; el Programa Internacional de Valoración de Estudiantes (PISA, 2000, 2009 y 2012) de la Organización para la Cooperación Económica y el Desarrollo (OCDE), entre otros estudios importantes, que demuestran que nuestra calidad de educación está por debajo del estándar de calidad: último lugar en el mundo. Por ejemplo, solo el 9 por ciento de los estudiantes de la población nacional de quinto de secundaria alcanzan el nivel suficiente; es decir, solo 1 de 10 alumnos que terminan secundaria ha logrado un aprendizaje más o menos aceptable.

Ante esta situación crítica que afronta la educación nacional, en los últimos años del presente siglo se ha suscitado un serio cuestionamiento al desempeño del profesorado de gestión pública, de parte de la opinión pública y los gobiernos de turno, pretendiéndose establecer una relación de causa efecto entre el bajo rendimiento académico de los estudiantes y la calidad de enseñanza brindada por los profesores, señalando al docente como el responsable de esta debacle. Sin embargo, tras un serio y profundo análisis e interpretación de los resultados de parte de los especialistas de entidades nacionales e internacionales, se ha llegado a la conclusión que el deterioro de la calidad de educación nacional es recurrente y no coyuntural, por lo tanto obedece a múltiples factores que intervienen en el proceso educativo, como la familia su constitución y su nivel cultural, la situación económica, política y social de nuestra sociedad, la nutrición, la infraestructura, entre otros, que determinan el deterioro de la calidad de educación, ensanchando las grandes brechas de inequidad.

Por lo que, a fin de subsanar los factores determinantes de la crisis educativa, se han adoptado desde el Ministerio de Educación y políticas del gobierno, diferentes medidas y programas, como la distribución gratuita de textos escolares, control de las horas efectivas de aprendizaje, utilización de las Tecnologías de Información y Comunicación (TIC), Plan Lector, olimpiadas de matemática, empleo de la metodología de proyectos educativos, 
capacitación y actualización permanente de docentes, etc. Asimismo, buscando mejorar la tarea docente se promulgó la Ley 29062 Ley de la Carrera Pública Magisterial que rige de manera paralela a la Ley 24029 Ley del Profesorado, la misma que otorga incrementos salariales sustantivos según el nivel magisterial y la carga horaria laboral, pero siguiendo una evaluación periódica del desempeño profesional.

En este contexto, dadas la evidencias irrecusables de la precarización del proceso de enseñanza y aprendizaje, constituye una tarea impostergable para los responsables de la labor educativa como el maestro de aula y para los que están comprometidos con el proceso educativo en una institución educativa, identificar las características de la relación director-docente-estudiante, y los factores determinantes de los bajos niveles de logros de aprendizaje de los estudiantes de nivel secundaria, y una vez identificado, impulsar y orientar la toma de decisiones para corregir estas falencias de tal manera que contribuya favorablemente en los logros de aprendizaje.

Queda absolutamente claro el papel central que cumple el docente en el proceso educativo, y como responsable de la gestión institucional le compete un rol importante al director, a través de sus actitudes y capacidades profesionales. Es decir, si bien existen factores asociados que influyen en los resultados descritos, es el factor institución educativa en quien recae la mayor responsabilidad del proceso educativo, por ello constituye el foco de atención de cualquier política de mejora.

El presente estudio se llevó a cabo en las instituciones educativas del nivel secundario de la RED $\mathrm{N}^{\circ}$ 09, del distrito de Villa María del Triunfo, Lima en el año 2014, y tiene como objetivo principal determinar la relación entre el liderazgo pedagógico del director y la evaluación del desempeño de los docentes, traducidos en resultados de aprendizajes en los estudiantes.

Entre los antecedentes del presente trabajo cabe mencionar la investigación realizada por Medina denominada Competencias del asesor técnico de Educación Físicadesde la perspectiva del liderazgo efectivo, cuyo objetivo fue determinar las competencias que posee el asesor técnico de Educación Física desde la perspectiva de liderazgo efectivo en las instituciones educativas del Municipio Escolar Maracaibo 04. El estudio constituye un aporte importante para la presente investigación por cuanto señala elementos teóricos referidos al liderazgo efectivo que debe ejercer todo gerente para llevar a cabo su función directiva, los cuales se han tomado como fundamento para las 
dimensiones e indicadores del Marco teórico.

Asimismo, se seleccionó el estudio realizado por Oviedo (2010) titulado Competencias intratégicas $\quad y$ liderazgo efectivo del supervisor en Educación Básica Primaria, el cual tuvo como propósito establecer la relación entre las variables planteadas, estudio realizado en la localidad el Municipio Escolar Maracaibo $\mathrm{N}^{\circ}$ 4. Se concluyó que las competencias intratégicas y el liderazgo efectivo de los supervisores educativos de Educación Básica Primaria están bien establecidas entre ellos, siendo puestas en práctica en su labor supervisora.

Además, se seleccionó el estudio de Pineda (2011) titulado Liderazgo efectivo y toma de decisiones en gerentes de Educación Inicial, cuyo propósito fue determinar la relación entre las variables planteadas en el Municipio Escolar Maracaibo $\mathrm{N}^{\circ} 3$ del estado Zulia. El estudio dio como resultado la correlación Rho Spearman de 0.819 lo cual indica una relación alta, estadísticamente significativa entre las variables objeto de estudio, concluyendo con ello que si incrementan los valores del liderazgo efectivo, repercute favorablemente en la toma de decisiones y viceversa.

En otro orden de ideas, se consideró la investigación de Artigas (2011) titulada Evaluación institucional y desempeño laboral de los docentes en los institutos de educación media general, cuyo propósito fue determinar la relación entre ambas variables en las instituciones mencionadas ubicadas en el Municipio Escolar Maracaibo $\mathrm{N}^{\circ} 5$ del estado Zulia. Se obtuvo como conclusión una correlación positiva significativa, al nivel de 0.01 , entre las variables, que permite colegir que, en la medida cómo se da el incremento de los valores de la evaluación institucional, aumentan también de forma media moderada alta los del desempeño laboral o viceversa en las instituciones objeto de estudio.

Se seleccionó también la investigación de Morales (2011) titulada Gerencia participativa y desempeño laboral del docente en educación media general que tuvo como objetivo general determinar la relación entre las variables en las instituciones del subsistema mencionado. En el análisis de los resultados se dedujo que tanto los directivos como los docentes siempre describen los factores básicos de la gerencia participativa e identifican las características del perfil del gerente participativo, mostrando debilidades en el desarrollo de la negociación y en las del gerente experto en humanidad, así como también las competencias, caracterizando los fundamentos profesionales del docente, 
manifestando

debilidad

en la comunicación efectiva, participación e incentivo.

También se seleccionó el estudio desarrollado por Pirela (2011) titulado Ambiente escolar y desempeño laboral docente en las instituciones de educación media general cuyo propósito fue determinar la relación entre las variables en el subsistema mencionado del Municipio Escolar San Francisco No ${ }^{\circ}$. La correlación indicó la existencia de una relación media estadísticamente significativa entre las variables, significando con ello que en la medida en que aumentan los valores del ambiente escolar, a su vez se incrementan los del desempeño laboral docente.

Espinoza (2010) en su importante estudio sobre el desempeño docente en una población constituida por 28 docentes de educación primaria de la institución educativa N $^{\circ} 20820$ de Huacho en el año 2009, que tuvo como objetivo determinar la influencia de la aplicación del Programa Nacional de Formación y Capacitación Permanente (PRONAFCAP) sobre el nivel de desempeño didáctico de los docentes, aplicó dos instrumentos, una encuesta de opinión para analizar el grado de aceptación y cumplimiento de la aplicación del plan de capacitación, y una lista de cotejo para el análisis del manejo cognitivo metodológico de los profesores en su desempeño didáctico en el aula. Los resultados arrojaron que existe un $60 \%$ de grado de aceptación de la aplicación del programa mientras que para el nivel de desempeño didáctico se observa un alto nivel de eficacia (66\%). Se concluye que la aplicación del programa permite elevar significativamente el nivel de desempeño didáctico de los docentes de educación primaria.

Quispe Quispe Primitivo (2011) de la Unidad de Postgrado de la Universidad Nacional Mayor de San Marcos, realizó la tesis: Relación entre el estilo de liderazgo del director y el desempeño docente en las instituciones educativas públicas del 2 do. Sector de Villa El Salvador de la UGEL 01 San Juan de Miraflores, en los años 2009 y 2010, en cuyas conclusiones se afirma que existe relación entre el estilo de liderazgo autoritario del director y el desempeño docente $(r=0,4675)$ en docentes $y$ $(r=0,4854)$ en alumnos como tal, este estilo de liderazgo es el que prevalece e interviene en el desempeño docente. Las puntuaciones logradas se han ubicado en el nivel regular (tabla 1 y 9) lo cual confirma la variable desempeño docente (tabla 4, 5, 6,8 y 16) por que la responsable en la toma de desicio9nes del director es vertical, lo demuestra el control a los 
docentes y el impedimento que las nuevas ideas de los docentes salgan a luz, imponiendo las suyas.

Sorados (2010) en una investigación descriptiva correlacional se propuso determinar la relación entre liderazgo y calidad de la gestión educativa. Se afirma con un $95 \%$ de probabilidad que el liderazgo de los directores se relaciona significativamente con la calidad de la gestión educativa de las instituciones educativas de la UGEL 03 - Lima en el 2009. Se asumió como población alumnos, docentes, trabajadores y directores de tres centros escolares, tomándose una muestra intencional de 20 sujetos. Los instrumentos del estudio fueron ficha de observación, encuesta y entrevista no estructurada. La prueba de correlación de Pearson arrojó un $r=.949$ siendo la dimensión pedagógica del liderazgo directivo la que más se relaciona con la calidad de la gestión educativa, se observó una correlación parcial significativa de $r=.937$. Por otra parte, la dimensión institucional es la que menos se relaciona con la gestión educativa pues se obtuvo una correlación parcial directa de $r=.461$.

Zarate Ramírez Dilma (2011) de la Unidad de Postgrado de la Universidad Nacional Mayor de San Marcos, realizó la tesis: Liderazgo directivo y el desempeño docente en instituciones educativas de primaria del distrito de Independencia, Lima. Siendo sus principales conclusiones, que existe una alta relación; el 95\% de los docentes están de acuerdo con el liderazgo directivo que presenta en las dimensiones gestión pedagógica, institucional y administrativa.

Otra investigación sobre liderazgo directivo fue llevada a cabo por Castillo (2010). En este estudio se buscó verificar la existencia de relación entre el liderazgo de la directora de la institución educativa inicial $N^{o} 87$ del Callao y el desempeño laboral de sus docentes. El trabajo de naturaleza descriptivo correlacional empleó como instrumentos de recogida de datos la encuesta y un cuestionario de autoevaluación docente. La autora menciona que la muestra incluyó un total de 110 padres de familia elegidos al azar entre las aulas de los niños de 3, 4 y 5 años de edad, y las 11 docentes que laboran en la institución. Las encuestas aplicadas arrojaron como resultado la no existencia de relación entre el estilo de liderazgo de la directora y el desempeño medio de las docentes.

Los objetivos de la presente investigación fueron:

\section{Objetivo General}

Determinar la influencia del liderazgo pedagógico del director en la evaluación del desempeño docente en las instituciones 
educativas del nivel secundario de la RED $\mathrm{N}^{\circ}$ 09, del distrito de Villa María del Triunfo, Lima 2014.

\section{Objetivos Específicos}

1. Identificar la influencia del liderazgo pedagógico del director en la planificación del trabajo docente en las instituciones educativas del nivel secundario de la RED $\mathrm{N}^{\circ}$ 09, del distrito de Villa María del Triunfo, Lima 2014.

2. Explicar la influencia del liderazgo pedagógico del director en los procesos de enseñanza docente en las instituciones educativas del nivel secundario de la RED $\mathrm{N}^{\circ}$ 09, del distrito de Villa María del Triunfo, Lima 2014.

\section{MATERIAL Y MÉTODO:}

El estudio de la presente investigación tiene dos variables; la variable $\mathrm{Y}$ (Liderazgo pedagógico del director) es el efecto y la causa es la variable $\mathrm{X}$ (Evaluación del desempeño docente).

Por el nivel de conocimientos, es explicativa porque da respuestas al por qué de la investigación y establece relaciones entre las variables para conocer la estructura y factores que intervienen en el proceso.

El diseño aplicable es correlacional explicativa, el cual permite establecer la relación entre las variables del estudio, se representa de la siguiente manera:

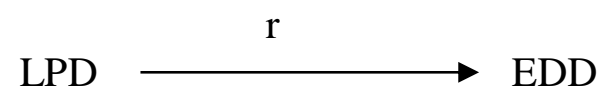

$\begin{array}{lcl}\operatorname{LPD}(\mathrm{X}) & = & \text { Liderazgo pedagógico del director } \\ \operatorname{EDD}(\mathrm{Y}) & = & \text { Evaluación del desempeño docente } \\ \mathrm{r} & = & \text { Correlación }\end{array}$

El instrumento que se aplicó para la integral del estudiante.

recolección de datos fue dos cuestionarios estructurados, para quienes forman parte del objeto de estudio, con el fin de recoger todos los datos necesarios que ayuden a establecer pautas y lineamientos para el análisis de Liderazgo pedagógico del director y por otro lado la formación
- Un cuestionario de Liderazgo pedagógico del director con preguntas cerradas (anónimo) para los docentes (Variable X).

- Un cuestionario de la Evaluación del desempeño docente del estudiante con 


\section{RESULTADOS Y DISCUSION:}

Gráfico $\mathbf{N}^{\mathbf{0}} \mathbf{1}$. Desempeño docente y las diferencias de medias

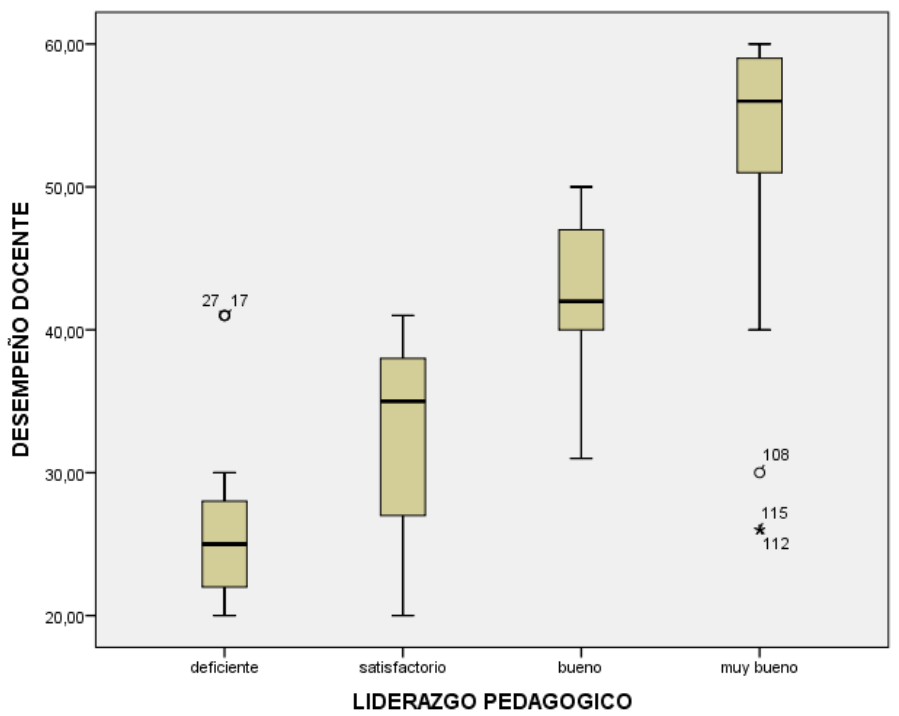

Se observa que el rango promedio del desempeño docente que presenta un deficiente liderazgo pedagógico es 27,08; el rango promedio de los que presentan un liderazgo satisfactorio es 49,24. El rango promedio de los que presentan un liderazgo bueno en el desempeño docente es 80,82 . El 108,86 posee un liderazgo muy bueno. Estos resultados nos indican que la mientras mejor sea el liderazgo pedagógico mayor será la media.

Gráfico $\mathbf{N}^{\mathbf{0}} \mathbf{2}$. Distribución porcentual de competencias en el desempeño docente

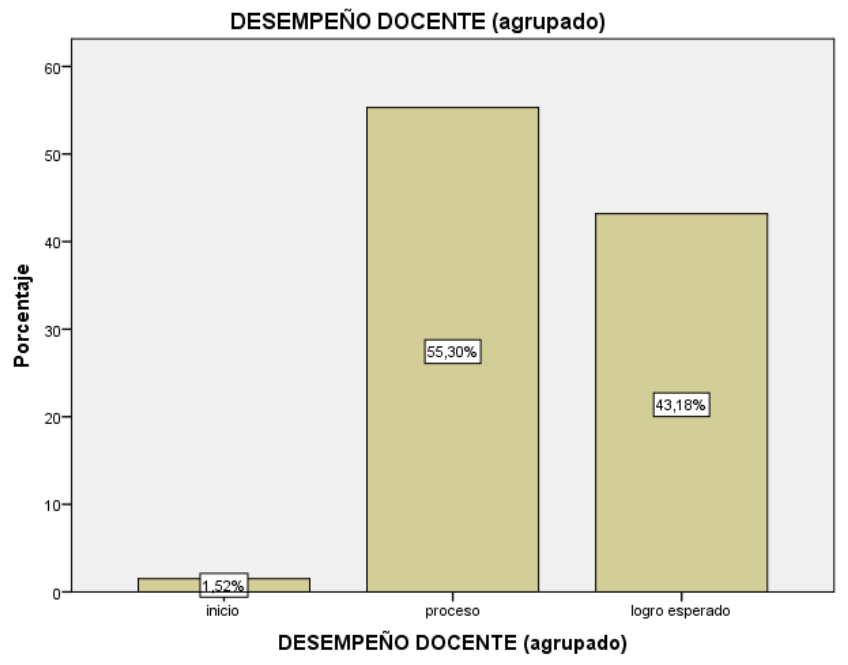


Se observa que el grupo mayoritario de docentes presentan un desempeño de competencias logradas en el nivel proceso con un 55,3\%, 57 docentes presentan un desempeño de logro esperado con 43,2 y con un 55,3\%. En el nivel inicio, se encuentra al $1,5 \%$ de docentes. Estos resultados nos indican que la mayoría de docentes alcanzan un nivel de logro esperado

Gráfico $\mathbf{N}^{\mathbf{0}}$ 3. Distribución porcentual del desempeño docente del liderazgo deficiente

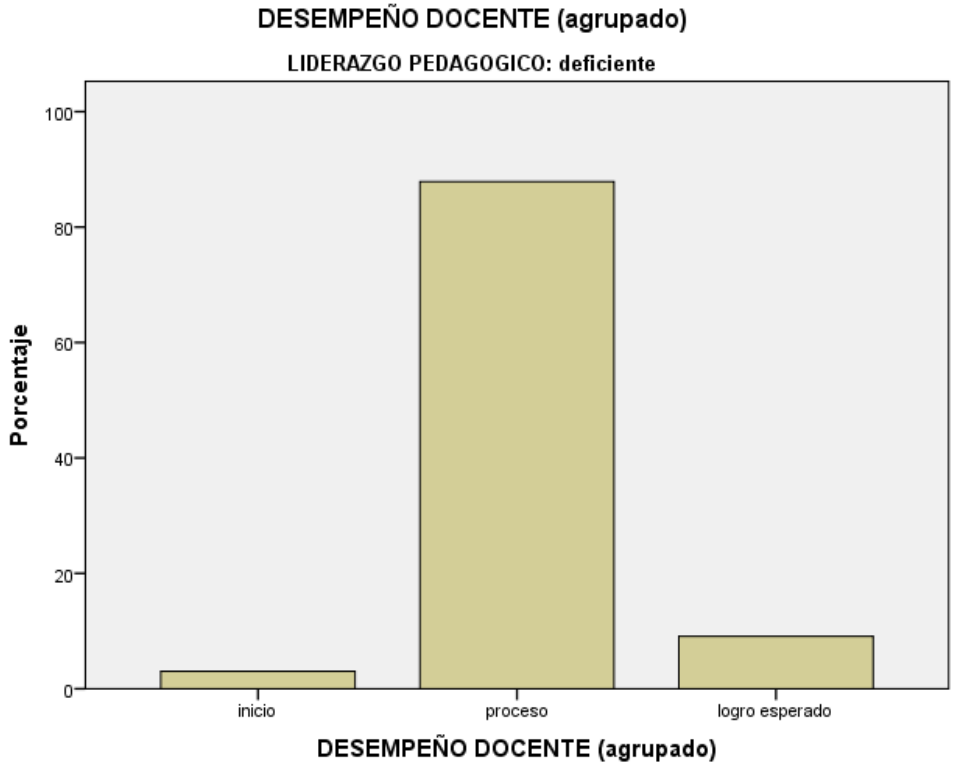

Se constata que el grupo mayoritario de docentes que presentan un liderazgo pedagógico deficiente presentan un desempeño en el nivel proceso con un $87,9 \%$, los docentes que presentan un desempeño de logro esperado representan al $9,1 \%$ y con un $3 \%$ se encuentran los docentes con un nivel de liderazgo deficiente en el nivel inicio. Estos resultados nos indican que la mayoría de docentes alcanzan un nivel en proceso. 
Gráfico $\mathbf{N}^{\mathbf{0}}$ 4. Desempeño docente del liderazgo satisfactorio

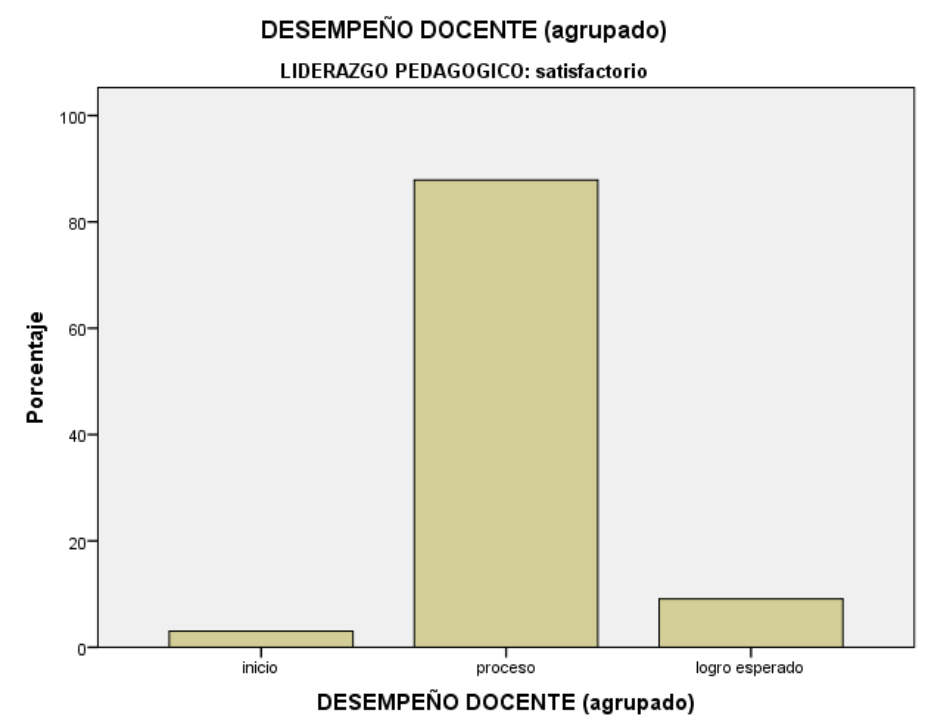

Se observa que el grupo mayoritario de al $9,1 \%$ y con un $3 \%$ se encuentran los docentes que presentan un liderazgo docentes con un nivel de liderazgo pedagógico satisfactorio presentan un satisfactorio en el nivel inicio. Estos desempeño en el nivel proceso con un resultados nos indican que la mayoría de $87,9 \%$, los docentes que presentan un docentes alcanzan un nivel en proceso. desempeño de logro esperado representan

Gráfico $\mathbf{N}^{\mathbf{0}}$ 6. Desempeño docente del liderazgo bueno

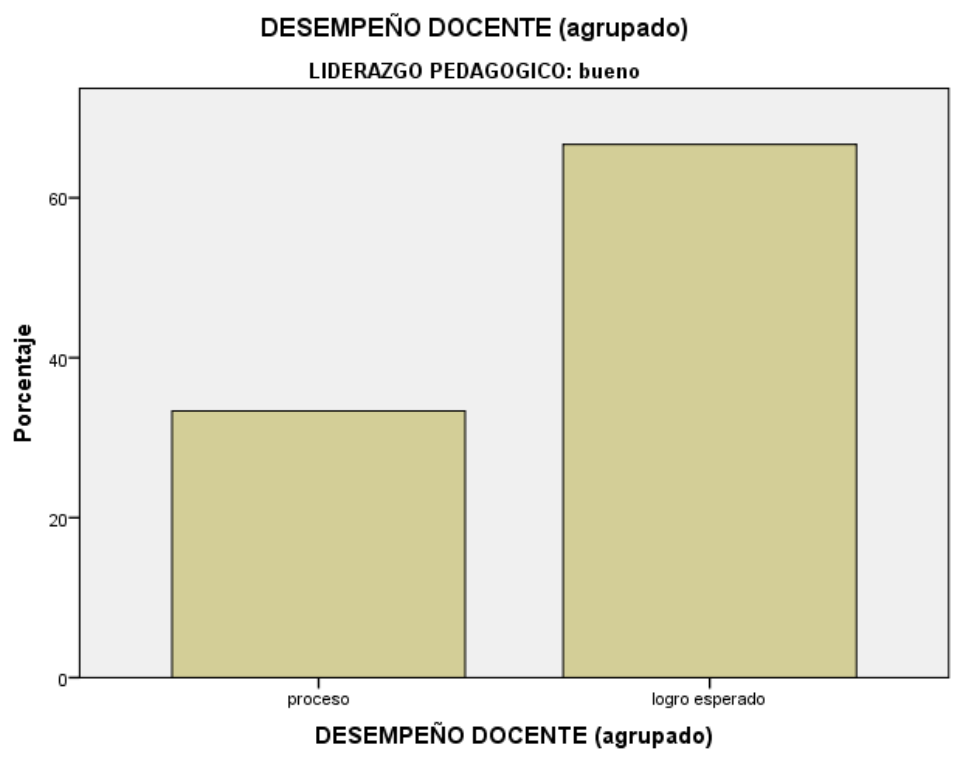


Se observa que el grupo mayoritario de docentes que presentan un liderazgo pedagógico bueno presentan un desempeño en el nivel logro esperado con un $66,7 \%$, los docentes que presentan un desempeño en proceso representan al 33,3\%. Estos resultados nos indican que la mayoría de docentes alcanzan un nivel de logro esperado.

Gráfico $\mathbf{N}^{\mathbf{0}} \mathbf{7}$. Desempeño docente del liderazgo muy bueno

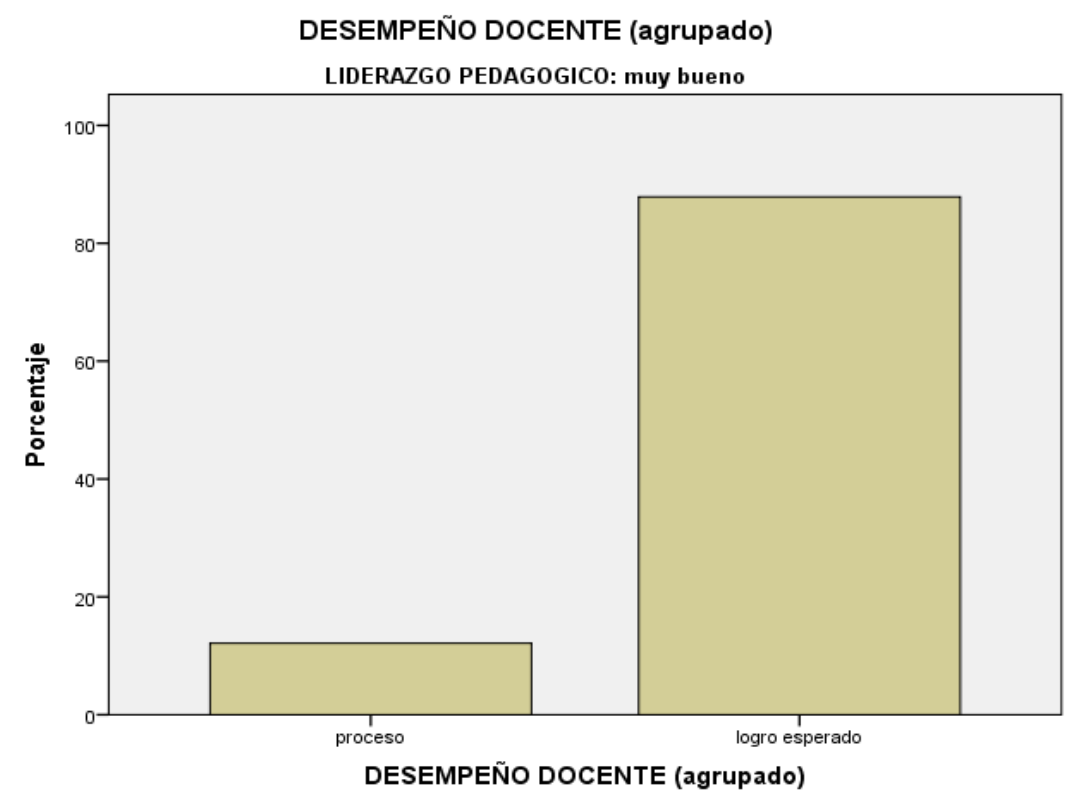

Se observa que el grupo mayoritario de docentes que presentan un liderazgo pedagógico muy bueno presentan un desempeño en el nivel logro esperado con un $87,9 \%$, los docentes que presentan un desempeño de logro esperado representan al $12,1 \%$. Estos resultados nos indican que la mayoría de docentes alcanzan un nivel de logro esperado.

\section{CONCLUSIONES}

\section{Primero}

Se ha demostrado que el liderazgo pedagógico del director influye en la evaluación del desempeño docente en las instituciones educativas del nivel secundario de la RED $\mathrm{N}^{\circ}$ 09, del distrito de Villa María del Triunfo, Lima 2014., según la Prueba de Kruskal-Wallises de 0,00 asociación de las variables y siendo altamente significativo. Además, según la regresión lineal el liderazgo influye en 42,4\% y se acepta la hipótesis: El liderazgo pedagógico del director influye en la evaluación del desempeño docente en las 
instituciones educativas del nivel secundario de la RED $\mathrm{N}^{\circ} 09$, del distrito de Villa María del Triunfo, Lima 2014.

\section{Segundo}

Se ha demostrado que el liderazgo pedagógico del director influye en la planificación del trabajo docente en las instituciones educativas del nivel secundario de la RED $\mathrm{N}^{\circ} 09$, del distrito de Villa María del Triunfo, Lima 2014, según la Prueba de Kruskal-Wallises de 0,00 asociación de las variables y siendo altamente significativo. Además, según la regresión lineal el liderazgo influye en $37,1 \%$ y se acepta la hipótesis: El liderazgo pedagógico del director influye en la planificación del trabajo docente en las instituciones educativas del nivel secundario de la RED $N^{\circ}$ 09, del distrito de Villa María del Triunfo, Lima 2014.

\section{Tercero}

Se ha demostrado que el liderazgo pedagógico del director influye en los procesos de enseñanza docente en las instituciones educativas del nivel secundario de la RED N ${ }^{\circ} 09$, del distrito de Villa María del Triunfo, Lima 2014., según la Prueba de Kruskal-Wallises de 0,00 asociación de las variables y siendo altamente significativo. Además, según la regresión lineal el liderazgo influye en $34,6 \%$ y se acepta la hipótesis: El liderazgo pedagógico del director influye en la conducción de los procesos de enseñanza en las instituciones educativas del nivel secundario de la RED $\mathrm{N}^{\circ}$ 09, del distrito de Villa María del Triunfo, Lima 2014.

\section{RECOMENDACIONES}

Primero

Se recomienda tener en consideración el nivel de liderazgo para mejorar el desempeño docente en las instituciones educativas.

Segundo

Se recomienda tener en consideración el nivel de liderazgo para mejorar la planificación del trabajo docente en las instituciones educativas.

Tercero

Se recomienda tener en consideración el nivel de liderazgo para mejorar los procesos de enseñanza docente en las instituciones educativas.

\section{REFERENCIAS BIBLIOGRAFICAS}

ARTIGAS, R. (2011) Evaluación institucional

y desempeño laboral de

los docentes en los institutos de educación media general. Trabajo de Grado. Universidad Rafael

Urdaneta. Maracaibo 
CASTILlO, V. (2010). Relación del liderazgo de la directora y el desempeño laboral de las docentes de la I.E.I. $\mathrm{n}^{\circ} 87$ Callao 2009. Lima, Universidad San Ignacio de Loyola, tesis de maestría.

ESPINOZA, J. (2010). Influencia de la aplicación del programa nacional de formación y capacitación permanente sobre el nivel de desempeño didáctico de los docentes de educación primaria de la Institución Educativa No 20820, Huacho. Lima: Universidad Nacional Mayor de San Marcos, tesis de maestría

MEDINA, V. (2010) Competencias del asesor técnico de Educación Física desde la perspectiva del liderazgo efectivo. Trabajo de Grado no publicado. Universidad del Zulia. Maracaibo

MORALES, R. (2011) Gerencia participativa y desempeño laboral del docente de educación media general. Trabajo de Grado no publicado. Universidad Rafael Urdaneta. Maracaibo

OVIEDO, J. (2010) Competencias estratégicas y liderazgo efectivo del supervisor en Educación Básica Primaria. Trabajo de Grado no Publicado. Universidad Rafael Urdaneta. Maracaibo

PINEDA, Y. (2011) Liderazgo efectivo y toma de decisiones en Gerentes de Educación Inicial. Trabajo de Grado no publicado. Universidad Rafael Urdaneta. Maracaibo

PIRELA, F. (2011) Ambiente escolar y desempeño laboral docente en las instituciones de educación media general. Trabajo de Grado no publicado. Universidad Rafael Urdaneta. Maracaibo

SORADOS, M. (2010). Influencia del liderazgo en la calidad de la gestión educativa. Lima: Universidad Nacional Mayor de San Marcos, tesis de maestría. 